\title{
Klinisch-morphologische Faktoren und der retroperitoneale Lymphknotenstatus beim (intraabdominalen) Stadium I des Ovarialkarzinoms
}

\begin{tabular}{|l|l|l|}
\hline E. & & Petru \\
\hline H. & & Pickel \\
\hline M. & & Lahousen \\
\hline M. & & Heydarfadai \\
\hline R. & & Winter \\
\hline
\end{tabular}

Geburtsh.-gynäkol. Univers.-Klinik Graz (Vorst.: Prof. Dr. R. Winter)

Dr. E. Petru, Geburtsh.-gynäkol. Univers.-Klinik, Auenbruggerplatz 14, A-8036 Graz

Es wurde retrospektiv die Beziehung zwischen klinisch-mor-phologischem Erscheinungsbild und dem retroperitonealen Lymphknotenstatus bei Patientinnen mit Ovarialkarzinom im Stadium I untersucht.

Patientinnen und Methode

Von 1980 bis Juli 1990 wurden insgesamt 100 auswertbare Patientinnen mit einem invasiven epithelialen Ovarialkarzinom im (intraabdominalen) Stadium I behandelt. Patientinnen mit Border-line-Tumoren wurden nicht berücksichtigt. 4 Patientinnen wurden ausschließlich einer unilateralen Adnexexstirpation und 6 Frauen nur einer beidseitigen Adnexexstirpation unterzogen. Bei weiteren 50 Patientinnen wurde eine Hysterektomie mit Adnexexstirpation beidseits \pm Omentektomie vorgenommen. Die verbleibenden 40 der 100 auswertbaren Patientinnen wurden neben einer Hysterektomie. beidseitiger Adnexexstirpation und Omentektomie auch einer sy-stematischen pelvinen Lymphadenektomie unterzogen; bei 21 Frauen wurde die Lymphadenektomie auf die Paraaortalregion ausgedehnt. Die Selektion von Patientinnen für eine Lymphadenektomie wurde vom Alter der Patientinnen, deren Allgemeinzustand und der intraoperativen Beurteilung durch den Anästhesisten beeinflußt.

Die Feststellung von statistisch signifikanten Unterschieden in den Patienten-Untergruppen erfolgte mit dem Fisher-Yates Test.

Ergebnisse

Die mittlere Anzahl der entfernten pelvinen und paraaortalen Lymphknoten betrug 38 bzw. 13. Von den 40 Patientinnen mit Lymphadenektomie wiesen 9 retroperitoneale Lymphknotenmetastasen auf (23\%). 7 der 19 Patientinnen, die nur einer pelvinen Lymphadenektomie unterzogen wurden, hatten positive Knoten.

Eine Patientin wies sowohl positive pelvine als auch paraaortale Lymphknoten auf; eine weitere hatte isoliert paraaortale Metastasen. Der Hauptanteil der pelvinen Metastasen beschränkte sich auf die Lnn. iliacae communes und Lnn. iliacae externae. Klinisch verdächtige Lymphknoten wurden intraoperativ bei 3 der 9 Patientinnen mit positiven Knoten registriert (33\%). Im Gegensatz dazu waren bei 7 der 31 Patientinnen mit negativen Lymphknoten diese klinisch auf metastatischen Befall suspekt (23 \%). 6 der 9 Patientinnen mit positiven Knoten wiesen einen 
einseitigen Tumor-befall der Ovarien auf. Bei einer von diesen wurden Metastasen in den kontralateralen pelvinen Lymphknoten gefunden. Vier der 16 Frauen unter 50 Jahren (25\%) bzw. 5 der 24 Frauen über 50 Jahren (21\%) hatten positive Lymphknoten (nicht signif.). Zwei der 11 Patientinnen im (intraabdominalen) Stadium la (18\%), eine der 3 Frauen im Stadium lb, und 6 der 26 Patientinnen im Stadium Ic (23\%) wiesen positive Lymphknoten auf (nicht signif.). Tumoren mit einem Maximaldurchmesser von $<5 \mathrm{~cm}(\mathrm{n}=7)$ wiesen die höchste Rate an positiven Lymphknoten auf ( $57 \%$ versus $15 \%$ bei den 33 Patientinnen mit Tumoren $>5 \mathrm{~cm}$; $\mathrm{p}=$ 0,01). Bei 8 der 21 serösen Zystadenokarzinome (38\%), einem der 11 endome-trioiden Karzinome (9\%), und keinem der 6 muzinösen Zystadenokarzinome und 2 mesonephrischen Karzinome wurden Lymphkno-tenmetastasen beobachtet. Während nur einer von 17 hochdifferenzierten Tumoren Lymphknotenmetastasen setzte (6\%), war dies bei 3 von 12 mittelgradig differenzierten (25\%) und 5 von 11 undifferenzierten Karzinomen der Fall (45\%) (p $=0,03)$. Bei 7 von 26 Patientinnen mit malignem Aszites bzw. maligner Peri-tonealzytologie (27\%) und 2 von 14 Frauen mit negativer Peri-tonealzytologie (14\%) wurden Lymphknotenmetastasen gefunden (nicht signif.). Während positive Lymphknoten bei 9 der 36 Patientinnen (25\%) ohne intraoperative Zystenruptur diagnostiziert wurden. trat dies in keinem der 4 Fälle mit Zystenruptur auf (nicht signif·)- Bei 14 Patientinnen mit Tumoren, die mit der Umgebung adhärent waren, wiesen 3 (21\%) Lymphknotenmetastasen auf. während dies bei 6 von 26 Patientinnen ohne Adhärenz der Fall war (21\%) (nicht signif.). Lymphknotenmetastasen bei Patientinnen ohne extrazystische Exkreszenzen wurden in 5 von 26 Fallen ge-funden (19\%), während positive Lymphknoten bei 4 der 14 Frauen (29\%) rait extrazystischen Exkreszenzen nachgewiesen wurden.

Diskussion und Schlußfolgerung

Es existieren nur wenige Daten zur Frage der Lymphadenektomie im Frühstadium des Ovarialkarzinoms. Es ist unklar, ob die Lymphadenektomie nur eine Staging-Maßnahme darstellt, ob sie als komplette Lymphadenektomie therapeutischen Zwecken dient, oder ob sie überhaupt durchgeführt werden soil. Viele Autoren und die FIGO haben jedoch umfangreiche Staging-Maßnahmen einschließlich der Lymphadenektomie beim Ovarialkarzinom gefordert. Klinisch-morphologische Faktoren wie Alter, FIGO-Substadium, Tu-morgröße, Peritonealzytologie, intraoperative Zystenruptur. Tumor-adhärenz oder extrazystische Exkreszenzen ergaben keinen sicheren Hinweis auf das Vorliegen von Lymphknotenmetastasen im (in-traabdominalen) Stadium I. Histologisch schlecht differenzierte und seröse Tumoren wiesen die höchste Rate an Lymphknotenmetastasen auf. Die intraoperative Klassifizierung des Differenzie-rungsgrades und der Histologie kann im Einzelfall Schwierigkeiten bereiten. Das hängt u. a. auch damit zusammen, daß das Schnell-schnittpräparat mit der endgültigen Histologie oft nur bedingt vergleichbar und damit für den Tumor nur eingeschränkt repräsentativ ist. Deshalb läßt sich nur schwer. wenn überhaupt. ein Risiko-kollektiv, bei dem im (intraabdominalen) Stadium 1 des Ovarialkarzinoms eine systematische Lymphadenektomie durchgeführt werden sollte, intraoperativ abgrenzen bzw. definieren.

Literatur

Knapp R. Friedman E: Aortic lymph node me-tastases in early ovarian cancer. Am J Obstet Gynecoi 1974; 119: 1013-1017.

Trimbos J 
Schueler A. van der Burg M: Watch and wait after careful surgical treatment und staging in welldifferentiated early ovarian cancer. Cancer 1991; 67: 597-602. 